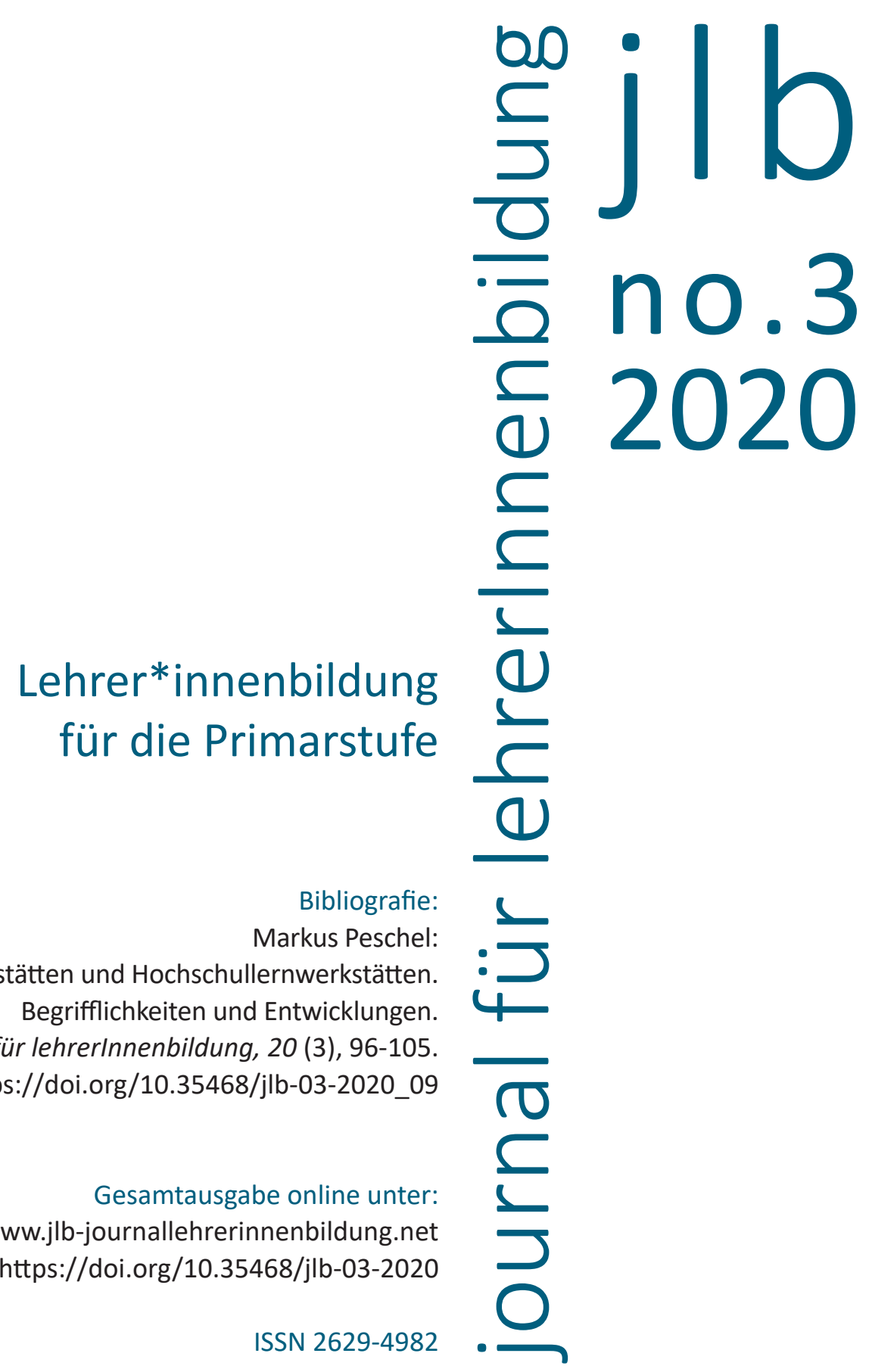


09

Markus Peschel

\section{Lernwerkstätten und Hochschullernwerkstätten. Begrifflichkeiten und Entwicklungen}


In den letzten Jahrzehnten sind Lernwerkstätten (LWS) und Hochschullernwerkstätten (HLWS) für die Kompetenzentwicklung angehender Lehrer*innen ${ }^{1}$ im deutschsprachigen Raum zu einem innovativen Ort für Pädagogik und Didaktik geworden, insbesondere weil sie einen wichtigen Handlungs- und Erfahrungsraum zur bildenden Auseinandersetzung darstellen. Dies betrifft vor allem das Zusammenspiel von pädagogischen, didaktischen und fachlichen Aspekten des Lehrens und Lernens. Durch die Arbeit von Interessensvereinigungen (VeLW, NeHle e. V. u. a.) oder von Forschungsaktivitäten an Universitäten und Hochschulen verbreiten sich die Theorie-Praxis-Konzeptionen (bisher) v. a. im deutschsprachigen Raum.

Der Beitrag zeichnet Entwicklungslinien und -tendenzen von Lernwerkstätten und Hochschullernwerkstätten im deutschsprachigen Raum nach. Dabei wird für eine sensible Begriffsverwendung und Begriffsdifferenzierung plädiert.

\section{Einführung}

Lernwerkstätten (LWS) an Grundschulen haben inzwischen eine lange Tradition in pädagogischen Kontexten, die sich zunehmend nicht nur in praktischen Konzepten, sondern auch in Ausbildungskontexten sowie theoretischen Konzeptionen und wissenschaftlicher Forschung widerspiegelt (vgl. für einen Überblick Ernst \& Wedekind, 1993; Hagstedt \& Krauth, 2014).

Deutlichen Anteil an der Entwicklung haben die langjährigen praktischen Erfahrungen bei der Umsetzung des Konzepts "Lernwerkstatt“ und der Tätigkeit des Begleitpersonals in LWS, häufig als "Lernwerkstattarbeit" (Schmude \& Wedekind, 2014, S. 110) oder "Lernwerkstattbegleitung" (Peschel \& Kihm, 2021 im Druck) bezeichnet. Verbreitung erfuhren diese Konzeptionen u. a. durch den Verbund Europäischer Lernwerkstätten, durch die Internationalen Fachtagungen der Hochschullernwerkstätten oder durch das Internationale Netzwerk der Hochschullernwerkstätten e. V. (NeHle).

Parallel dazu entwickelte sich die Ausbildung für und in Lernwerkstätten, die an Universitäten und Hochschulen - als Hochschullernwerk-

1 Aufgrund des Leser*innenbezugs wird in diesem Beitrag auf LWS und HLWS mit Grundschulbezug rekurriert, wohlwissend, dass für (angehende) Pädagog*innen und LWS an Kindergärten etc. diese Ausführungen Äquivalenz finden. 
stätten (siehe unten) - die Konzeptionen innovativen pädagogischdidaktischen Handelns in Lernwerkstätten thematisieren und vermitteln. Hochschullernwerkstätten (HLWS) wurden, diesem Verständnis entsprechend, als Orte der Ausbildung von angehenden Lehrkräften implementiert. Die Akteur*innen und Zielgruppen in HLWS sind folglich primär Studierende und erst sekundär Schüler*innen, Lehrkräfte, Eltern u. a.m. (vgl. Kihm \& Peschel, 2017, 2020; Franz, 2012; Graf \& Kekeritz, 2016). „In HLWS geht es in erster Linie um die Ausbildung von Studierenden und erst in zweiter Linie um begleitende Schülerbesuche - entweder als Teil der Ausbildung der Studierenden oder als zusätzliches Angebot neben dem ,Hauptgeschäft' Lehrer*innenbildung" (Peschel \& Kihm, 2021 im Druck).

Weiterhin ist zunehmend auch der Einfluss von - meist grundschulorientierten - Fachdidaktiken für die Vermittlungspositionen in HLWS einzubeziehen, da LWS zwar aus einer pädagogischen Historie entstanden sind, Lernprozesse aber zunehmend fachdidaktisch differenziert und definiert werden (vgl. Peschel \& Kelkel, 2018a).

Gemeinsam ist den meisten konzeptionellen Ansätzen in Lernwerkstattzusammenhängen, dass das Lernen der Schüler*innen bzw. allgemein der Lernenden im Mittelpunkt der Aktivitäten steht. Dies allein unter dem Aspekt der Öffnung von Lernsituationen zu sehen und Zuordnungen wie "offen gleich Lernwerkstatt" und "geschlossen gleich Unterricht "vorzunehmen, wird den ausdifferenzierten Ansätzen nicht gerecht und polarisiert nach Hildebrandt, Peschel und Weißhaupt (2014) Positionen, die aber grundlegend für alle Lehr-Lern-Prozesse unabhängig von der jeweiligen Institution (ob HLWS, LWS oder Schule) zu verstehen sind.

\section{Lernwerkstatt (LWS)}

Lernwerkstatt (LWS) hat sich als Begriff für die zumeist räumliche Verortung eines Lernens herausgebildet, das von üblichen Formen der Vermittlung abkehrt und das Lernen der Kinder im Sinne von "Selbstlernkonstruktionen" (Peschel, 2016, S. 123) in den Mittelpunkt der Auseinandersetzung rückt - daher LERNwerkstatt. Dies wurde u. a. durch konzeptionelle Grundlegungen von Hagstedt (1990), Ernst und Wedekind (1993) oder VeLW (2009) verbreitet. Allerdings ist LWS kein geschützter Begriff und es tummeln sich vielfältige Spielarten unter 
der Bezeichnung. Verwendungen des Begriffs LWS beziehen sich z. B. auf Lernangebote, mediale Konzeptionen oder Arbeitsblattsammlungen. Der Bezug geht hier eventuell auf den Werkstattbegriff nach Jürgen Reichen zurück, beinhaltet jedoch nicht das pädagogischdidaktische Konstrukt im oben genannten Sinne. Insofern muss der „Umbrella-Begriff" (Schmude \& Wedekind, 2014, S. 108) sorgsam definiert, erläutert und konkretisiert werden.

Der Bezug auf das "Werk" in einer LernWERKstatt wird ebenso unterschiedlich interpretiert. Auch wenn das Lernen vielfach im Hinblick auf ein "als Produkt ausgerichtetes, vollendetes Werk" (Franz, 2012, S. 22) in den Mittelpunkt gerückt wird, ist das "Werk" in LWS zumeist nicht klar definiert. Die Bezüge auf das Lernen und die Stätte (LERNwerkSTATT) dominieren nach Peschel und Kelkel (2018a) zumeist den fachlichen oder fachdidaktischen Bezug.

Schmude und Wedekind (2014) betonen, dass der Begriff LernwerkSTATT den Raum als Stätte beinhaltet. Dieser ist zumeist als materieller und physischer Raum verortet (vgl. Müller-Naendrup, 1997). Dabei wird der Raumbegriff in verschiedenen pädagogisch-didaktischen Zusammenhängen interpretiert (vgl. Wedekind, 2006; Kaiser, 2016), nach Peschel und Kelkel (2018a, 2018b) aber seltener aus fachlicher oder fachdidaktischer Sichtweise. Auch virtuelle Räume bzw. digitale Angebote werden nach Dörrenbächer, Hart und Perels (2018) als LWS bezeichnet.

Neuere Differenzierungen erlauben es, Aspekte der LWS als Raum und der Lernwerkstattarbeit als Tätigkeit in LWS deutlicher zu diskutieren (AG Begriffsbestimmung - NeHle, 2020). Die Ausarbeitungen seitens NeHle (ebd.) zeigen, dass eine sensible und sorgfältige Klärung, was die Grundlage oder Festschreibung einer LWS oder HLWS ausmacht, notwendig ist.

\section{Hochschullernwerkstätten (HLWS)}

Hochschullernwerkstätten (HLWS) sind nach Schude (2016) Ausbildungsorte an einer Universität oder Hochschule - zumeist in der Pädagog*innenbildung. Diese sind meist als physischer Raum - teils auch als Studienwerkstätten bezeichnet - strukturell in die Ausbildung angehender Lehrer*innen eingebunden (vgl. Baar, Feindt \& Trostmann, 2019). Peschel und Kihm (2021 im Druck) betonen, dass in die- 
sem Rahmen dort auch Forschung stattfindet bzw. die Studierenden frühzeitig in Forschungsprojekte eingebunden sind.

\section{Studieren in HLWS}

In HLWS werden Ideen und Konzepte von LWS vermittelt und die Begleitung von Lernenden (häufig als "Lernwerkstattarbeit" bezeichnet) theoretisch und praktisch in einem konsequenten „Didaktischen Labor" der Hochschule vermittelt.

HLWS grenzen sich - ebenso wie LWS - i. d. R. von instruktionistischen Lehr-Lern-Formaten ab (vgl. Müller-Naendrup, 1997; Franz, 2012; Kaiser, 2016) und entwickeln - aufbauend auf einem konstruktivistischen Lernverständnis - eine „innovative Didaktik“ (Rumpf, 2016, S. 76; Baar et al., 2019, S. 11): Der pädagogisch-didaktische Anspruch von HLWS und der zu „,vermittelnden“ Didaktik ist es, dass Studierende durch Eigenerfahrung lernen, wie sie in der „Rolle Schüler*innen“ (Peschel \& Kihm, 2021 im Druck) konstruktiv und selbstbestimmt lernen, indem sie frei tätig sind (vgl. Gruhn \& Müller-Naendrup, 2017). Dazu sollen Studierende in HLWS "Selbstlernkonstruktionen nachgehen und sich Lerninhalte [...] selbst erschließen" (Peschel, 2016, S. 123) - dies zunehmend mittels eigener Lernwege und in Auseinandersetzung sowie Reflexion des eigenen Lernverständnisses und mit eigenen, selbst gesetzten Lernzielen (vgl. Hagstedt, 2016; Peschel, 2014).

Dieser Anspruch an das Lernen und die Bezüge zu Lerntheorien zeigen, Hildebrandt et al. (2014) folgend, dass der Konstruktivismus in HLWS selbstreflexiv und theoretisch wie praktisch vermittelt wird. Die Auseinandersetzung zwischen Lerner und Lerngegenstand wird selbst erfahren und vermittelt praxisgerecht lerntheoretische Bezüge wie „kindorientiert", „selbstbestimmt" oder „eigenaktiv".

„In der vorrangig in Lernwerkstätten realisierten Lernwerkstattarbeit in pädagogischen Studiengängen an Hochschulen geht es vor allem darum, das Lernen zu lernen, entdeckend Dingen auf den Grund zu gehen und auf der Grundlage der dabei gemachten Erfahrungen didaktische Implikationen für die eigene pädagogische Arbeit abzuleiten." (Schmude \& Wedekind, 2019, S. 40f.).

Anders als z. B. Schülerlabore, die als Lehr-Lern-Labore (Schülerlabor ${ }^{L}$ ) ebenfalls in die Lehrerbildung wirken (vgl. Priemer \& Roth, 2020), konkretisieren HLWS zumeist eine pädagogische oder didaktische Intention und fokussieren daher lernbezogene Inhalten und Lehr- bzw. Lern- 
methoden. Die Argumente für die curriculare Einbindung von HLWS in pädagogische Ausbildungsgänge lassen sich somit nach Peschel (2016) vornehmlich auf pädagogischer und didaktischer Ebene verorten; historisch gesehen sind die HLWS meist aus einem überfachlichen Interesse oder mit einem überfachlichen bzw. Grundschulbezug entstanden (vgl. Rumpf, 2016):

In HLWS werden universitäre Lehre und innovative Didaktik im Ausbildungsfeld Schule/Kindergarten unmittelbar verknüpft und entfalten daher ein hohes Potenzial für eine Theorie-Praxis-Verzahnung (vgl. Stadler-Altmann, 2019; Schöps, Rumpf \& Kramer, 2019; Franz, 2012). Studierende können direkt in Aspekte der Lernwerkstattbegleitung, in Reflexionen über Lehren und Lernen, in Prozesse der Veränderung oder Aushandlung von Rollenverständnissen und in die Erforschung solcher Prozesse eingebunden werden (vgl. Rumpf, 2016; Kelkel \& Peschel, 2019). Um den damit verbundenen, gemäßigten Konstruktivismus' nicht nur theoretisch kennenzulernen, sondern als nutzbare und ggf. sinnvolle lerntheoretische Grundlage für Lernarrangements zu begreifen, fordert Huber (2004), dass die Studierenden entsprechende Lernarrangements planen, einsetzen und - durch eigene Forschung, ggf. in Qualifikationsarbeiten - beforschen müssen.

\section{Forschen in Hochschullernwerkstätten}

Spezifische Aspekte, wie die Arbeit und das Lernen von Studierenden untereinander (in Seminarkontexten), aber auch die Arbeit in HLWS mit Kindern (in einem didaktischen Laborkontext), können so forschend in den Blick genommen werden. In diesem Rahmen erfolgt in HLWS neben Lehre auch Forschung, um Prozesse der Verknüpfung von universitärer Lehre und Forschung unmittelbar anzubahnen.

Diese Möglichkeiten der HLWS, Studierende forschend in einem schulorientierten Lehr-Lern-Setting einzubinden und eine Erforschung im Rahmen der Lehre ihrer eigenen Lehrer*innenausbildung praktizieren zu lassen, ist vor allem für die Erkenntnis der Forschungsbasiertheit von Lehre an Hochschulen ein wichtiger Baustein. Zudem hilft es Studierenden nach Rumpf (2016) und Stadler-Altmann (2019), ihr zukünftiges Berufsfeld vorzubereiten und als Praxis theoretisch fundiert verstehen zu können.

Um „Selbstlernkonstruktionen“ (Peschel, 2016, S. 123) bzw. Lehr-LernProzesse in HLWS aber verstehen zu können, ist es nach Huber (2004) notwendig, solche Forschungsprozesse zu unterstützen, die: 
- individuelle Forschungsschwerpunkte wählen

- passende Forschungsfragen generieren

- Forschungsvorhaben (z. B. für ihre Qualifikationsarbeiten) planen

- sich mit ihren Erhebungsverfahren an der Forschungsfrage orientieren

- in den HLWS selbständig Untersuchungen durchführen

- innovative Lehr-Lern-Szenarien entwickeln und in pädagogischen Realsituationen erproben

- Ergebnisse in Diskussionsrunden, wissenschaftlichen Kolloquien o. ä. vorstellen, interpretieren, diskutieren - und auch „verteidigen“

- Forschungsarbeiten schreiben.

Die Erprobung und Erforschung erlaubt Interpretationen im Hinblick auf die Wirkung einzelner Personengruppen und ihrer Rollen sowie der entsprechenden Wechselwirkungen untereinander (z. B. Interaktionen, vgl. Diener \& Peschel, 2019). Die Entwicklung der eigenen Rolle als „Lernbegleitung“ ist ein sensibles Feld bei den handelnden Studierenden; die geforderte „innovative Didaktik“ - im Lehr-Lern-Bezug auf vermittelnde (fachbezogene) Inhalte - kann sich insbesondere durch die Implementierung einer handlungsfähigen Rolle als "Lernbegleitung" erschließen.

Zentral ist dabei m. E. die Einbindung und Reflexion der jeweiligen Lehr-Lern-Forschungen und die Auswirkungen auf Lehr-Lern-Situationen in HLWS, einschließlich Fragen, wie sich z. B. eine Öffnung - und damit einhergehend entsprechende Aufgabenkonstruktionen und eine veränderte Lehrer*innenrolle (vgl. Kihm \& Peschel, 2017; Diener \& Peschel, 2019) - auf den Lern- und Erkenntnisprozess auswirkt. Durch reflexive Bezüge lernen Studierende, sich in ihrer Rolle als Lernbegleitung zurückzunehmen (vgl. Franz, 2012; Peschel, 2016; Kaiser, 2016) und erfahren Konstruktivismus nicht als „bloße" Reduktion instruktionaler Anteile, sondern als eigen-konstruktive Leistung von Kindern (vgl. Reich, 2012; Arnold, 2012).

\section{Fazit}

Nachdem die Begrifflichkeiten im Zusammenhang mit LWS und HLWS sowie die Implikationen und Mehrdeutigkeiten dieser Begrifflichkeiten - expliziert und aktuelle Entwicklungen skizziert wurden, ist es m. E. dringend erforderlich, eine Lernwerkstattdidaktik zu etablieren, 
die nicht nur pädagogisch orientiert ist, sondern auch die beinhalteten Sachauseinandersetzungen (z. B. Experimentieren, Schriftspracherwerb und Schriftlichkeit, Geometrie, Arithmetik u.v. m.) in einem eigenen Verständnis entwickelt - orientiert an den Grundschuldidaktiken der Fächer.

Dies erfordertm. E. eineneue Fachdidaktik, die Fach-und Lernwerkstattzusammenhänge zusammenfasst und in einer doppelten Funktionalität - angelehnt an den Didaktischen Doppeldecker (Wahl, 1991) Aspekte von Lernwerkstattpädagogik und Fachdidaktik zusammenbringt. Der Ort dieser Vermittlung - und vor allem der Forschung diesbezüglich - sind Hochschulen, konkret: HLWS, die die o. g. Positionen einer theoretischen und praktischen Funktionalität mit Studierenden grundlegen. Dass dabei eine Theorie(weiter)entwicklung und entsprechende empirische Forschungen notwendig sind, scheint offensichtlich, haben doch LWS das Potenzial gezeigt, innovative Pädagogik und Didaktik als gute Praxis zu etablieren.

\section{Literatur}

AG Begriffsbestimmung - NeHle (2020). Internationales Netzwerk der Hochschullernwerkstätten e. V. - NeHle - ein Arbeitspapier der AG „Begriffsbestimmung Hochschullernwerkstatt" zum aktuellen Stand des Arbeitsprozesses. In U. Stadler-Altmann, S. Schumacher, E. A. Emili \& E. Dalla Torre (Hrsg.), Spielen, Lernen, Arbeiten in Lernwerkstätten. Facetten der Kooperation und Kollaboration. Bad Heilbrunn: Klinkhardt.

Arnold, R. (2012). „Ich lerne, also bin ich". Eine systemisch-konstruktivistische Didaktik. Heidelberg: Carl Auer.

Baar, R., Feindt, A. \& Trostmann, S. (Hrsg.). (2019). Struktur und Handlung in Lernwerkstätten- Hochschuldidaktische Räume zwischen Einschränkung und Ermöglichung. Bad Heilbrunn: Klinkhardt.

Diener, J. \& Peschel, M. (2019). Lehrerhandeln im Grundschullabor für Offenes Experimentieren. In M. Peschel \& U. Carle (Hrsg.), Praxisforschung Sachunterricht (S. 11-34). Baltmannsweiler: Schneider Verlag Hohengehren.

Dörrenbächer, L., Hart, I. \& Perels, F. (2018). Konzeption einer überfachlichen Lernwerkstatt für Lehramtsstudierende zur Förderung des selbstregulierten Lernens. In M. Peschel \& M. Kelkel (Hrsg.), Fachlichkeit in Lernwerkstätten (S. 122-137). Bad Heilbrunn: Klinkhardt.

Ernst, K. \& Wedekind, H. (Hrsg.). (1993). Lernwerkstätten in der Bundesrepublik Deutschland und Österreich: Eine Dokumentation. Beiträge zur Reform der Grundschule. Frankfurt am Main: Grundschulverband - Arbeitskreis Grundschule.

Franz, E.-K. (2012). Lernwerkstätten an Hochschulen: Orte der gemeinsamen Qualifikation von Studierenden, pädagogischen Fachkräften des Elementarbereichs und 
Lehrkräften der Primarstufe. Frankfurt am Main: Peter Lang GmbH, Internationaler Verlag der Wissenschaften.

Graf, U. \& Kekeritz, M. (2016). Über eine akzeptierende und reflexive Dialogkultur im Lernwerksattskontext. Wie ein Kind sich in der Perspektive der Lernbegleiter*innen verändert. In C. Schmude \& H. Wedekind (Hrsg.), Lernwerkstätten an Hochschulen (S. 147-171). Bad Heilbrunn: Klinkhardt.

Gruhn, A. \& Müller-Naendrup, B. (2017). „Theoretische Kreativität" in Hochschullernwerkstätten - ein Plädoyer. In M. Kekeritz, U. Graf, A. Brenne, M. Fiegert, E. Gläser \& I. Kunze (Hrsg.), Lernwerkstattsarbeit als Prinzip (S. 100-111). Bad Heilbrunn: Klinkhardt.

Hagstedt, H. (1990). Lernwerkstätten - neue Lebensräume für LehrerInnen? Päd. Extra \& demokratische Erziehung, 3, 18-19.

Hagstedt, H. (2016). Lernen im Selbstversuch. In S. Schude, D. Bosse \& J. Klusmeyer (Hrsg.), Studienwerkstätten in der Lehrerbildung. Theoriebasierte Praxislernorte an der Hochschule (S. 27-36). Wiesbaden: Springer VS.

Hagstedt, H. \& Krauth, I. M. (Hrsg.). (2014). Lernwerkstätten. Potenziale für Schulen von morgen. Beiträge zur Reform der Grundschule. Frankfurt am Main: Grundschulverband e. V.

Hildebrandt, E., Peschel, M. \& Weißhaupt, M. (Hrsg.). (2014). Lernen zwischen freiem und instruiertem Tätigsein. Bad Heilbrunn: Klinkhardt.

Huber, L. (2004). Forschendes Lernen. 10 Thesen zum Verhältnis von Forschung und Lehre aus der Perspektive des Studiums. Die Hochschule: Journal für Wissenschaft und Bildung, 13 (2), 29-49.

Kaiser, L. S. (2016). Lernwerkstattarbeit in kindheitspädagogischen Studiengängen. Empirische Studien zur Theorie-Praxis-Verknüpfung. München: kopaed.

Kelkel, M. \& Peschel, M. (2019). Lernwerkstätten und Schülerlabore - Unterschiedliche Konzepte, ein Verbund. In R. Baar, A. Feindt \& S. Trostmann (Hrsg.), Struktur und Handlung in Lernwerkstätten (S. 185-189). Bad Heilbrunn: Klinkhardt.

Kihm, P. \& Peschel, M. (2017). Interaktion und Kommunikation beim Experimentieren von Kindern - Eine Untersuchung über interaktions- und kommunikationsförderliche Aufgabenformate. In M. Peschel \& U. Carle (Hrsg.), Forschung für die Praxis. Beiträge zur Reform der Grundschule (S. 66-80). Frankfurt am Main: Grundschulverband e. V.

Kihm, P. \& Peschel, M. (2020). Einflüsse von Aushandlungs- und Interaktionsprozessen auf Lernwerkstattarbeit. In U. Stadler-Altmann, S. Schumacher, E. A. Emili \& E. Dalla Torre (Hrsg.), Spielen, Lernen, Arbeiten in Lernwerkstätten (S. 87-99). Bad Heilbrunn: Klinkhardt.

Müller-Naendrup, B. (1997). Lernwerkstätten an Hochschulen: Ein Beitrag zur Reform der Primarstufenlehrerbildung. New York: Peter Lang $\mathrm{GmbH}$, Internationaler Verlag der Wissenschaften.

Peschel, M. (2014). Vom instruierten zum Freien Forschen Selbstbestimmungskonzepte im GOFEX. In E. Hildebrandt, M. Peschel \& M. Weißhaupt (Hrsg.), Lernen zwischen freiem und instruiertem Tätigsein (S. 67-79). Bad Heilbrunn: Klinkhardt.

Peschel, M. (2016). Offenes Experimentieren - Individuelles Lernen. Aufgaben in Lernwerkstätten. In H. Hahn, I. Esslinger-Hinz \& A. Panagiotopoulou (Hrsg.), Paradigmen und Paradigmenwechsel in der Grundschulpädagogik (S. 120-129). Baltmannsweiler: Schneider-Verlag Hohengehren.

Peschel, M. \& Kelkel, M. (Hrsg.). (2018a). Fachlichkeit in Lernwerkstätten - Kind und Sache in Lernwerkstätten. Bad Heilbrunn: Klinkhardt. 
Peschel, M. \& Kelkel, M. (2018b). Potenziale von Lernwerkstätten zur Vermittlung von Handlungskompetenzen angehender Lehrkräfte. GDSU Journal 8, 31-46.

Peschel, M. \& Kihm, P. (2021 im Druck). Rollenverständnisse und Rollenaushandlungen in Hochschullernwerkstätten. In M. Schöps, D. Rumpf, K. Kramer \& S. Winter (Hrsg.), Hochschulwerkstätten - Elemente von Hochschulentwicklung? Bad Heilbrunn: Klinkhardt.

Priemer, B. \& Roth, J. (2020). Lehr-Lern-Labore. Wiesbaden: Springer Spektrum.

Reich, K. (2012). Konstruktivistische Didaktik: Das Lehr- und Studienbuch. Weinheim: Beltz.

Rumpf, D. (2016). Forschendes Lernen und Forschen lernen in Hochschullernwerkstätten. In S. Schude, D. Bosse, J. Klusmeyer (Hrsg.), Studienwerkstätten in der Lehrerbildung (S. 73-85). Wiesbaden: Springer VS.

Schmude, C. \& Wedekind, H. (2014). Lernwerkstätten an Hochschulen. Orte einer inklusiven Pädagogik. In E. Hildebrandt, M. Peschel \& M. Weißhaupt (Hrsg.), Lernen zwischen freiem und instruiertem Tätigsein (S. 103-122). Bad Heilbrunn: Klinkhardt.

Schmude, C. \& Wedekind, H. (2019). Lernwerkstatt(arbeit) zwischen pädagogischem Anspruch und strukturellen Rahmenbedingungen. In R. Baar, A. Feindt \& S. Trostmann (Hrsg.), Struktur und Handlung in Lernwerkstätten (S. 40-50). Bad Heilbrunn: Klinkhardt.

Schöps, M., Rumpf, D. \& Kramer, K. (2019). Lernwerkstatt(arbeit) zwischen pädagogischem Anspruch und strukturellen Rahmenbedingungen. In S. Tänzer, M. Godau, M. Berger \& G. Mannhaupt (Hrsg.), Perspektiven auf Hochschullernwerkstätten in der Lehrerbildung (S. 19-32). Bad Heilbrunn: Klinkhardt.

Schude, S. (2016). Studienwerkstätten als bedeutsame Lernumgebung in Hochschule und Schule. In S. Schude, D. Bosse \& J. Klusmeyer (Hrsg.), Studienwerkstätten in der Lehrerbildung (S. 9-26). Wiesbaden: Springer VS.

Stadler-Altmann, U. (2019). EduSpace Lernwerkstatt als Verknüpfungsraum zwischen Praktikum und universitärer Lehre. In R. Baar, A. Feindt \& S. Trostmann (Hrsg.), Struktur und Handlung in Lernwerkstätten (S. 201-214). Bad Heilbrunn: Klinkhardt.

VeLW [Verbund europäischer Lernwerkstätten e. V.] (2009). Positionspapier zu Qualitätsmerkmalen von Lernwerkstätten und Lernwerksattsarbeit. Bad Urach.

Wahl, D. (1991). Handeln unter Druck. Der weite Weg vom Wissen zum Handeln bei Lehrern, Hochschullehrern und Erwachsenenbildnern. Tübingen: Deutscher Studien-Verlag.

Wedekind, H. (2006). Didaktische Räume - Lernwerkstätten. Orte einer basisorientierten Bildungsinnovation. Gruppe \& Spiel, (4), 9-12.

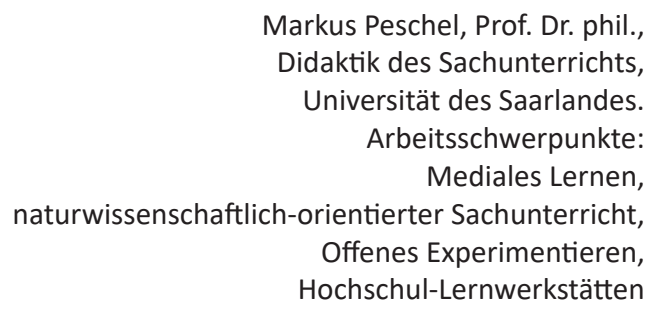

markus.peschel@uni-saarland.de

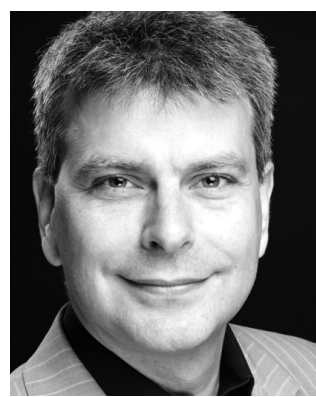

